\title{
8 \\ Eigenpsychisches und Fremdpsychisches: Rudolf Carnaps Verhältnis zur Psychologie zwischen 1928 und 1932
}

\author{
Uljana Feest
}

\subsection{Einleitung}

Carnaps Werk zwischen den späten 1920er- und frühen 1930er-Jahren nimmt verschiedentlich auf Begrifflichkeiten und Debatten der philosophischen und experimentellen Psychologie seiner Zeit Bezug. Diese Bezugnahmen sind jedoch nicht immer konsistent oder explizit. Beispielsweise bedient er sich sowohl im Aufbau (1928) als auch in seinen Ausführungen zur Psychologie in einer physikalischen Sprache (1932) einiger Grundannahmen der experimentellen Psychologie (speziell der Psychophysik und der psychophysikalisch vorgehenden Gestaltpsychologie), führt dies jedoch nicht konsequent zu Ende (vgl. Feest 2007, 2017). So finden sich etwa in Carnaps Aufbau trotz seines affirmativen Bezuges auf die Gestaltpsychologie u. a. psychologische Annahmen, die denen der Gestaltpsychologie widersprechen. Umgekehrt macht er in seinen Arbeiten zur Psychologie in physikalischer Sprache aber Anleihen bei der psychophysischen Forschung (wie sie auch von der Gestaltpsychologie praktiziert wurde), erkennt dies allerdings nicht explizit an, sondern betont im Gegenteil seine Kritik an bestimmten Forschungspraktiken der Psychologie.

In diesem Aufsatz gehe ich der Frage nach, wie Carnaps Bezugnahme auf die und Abgrenzung von der - Psychologie einzuschätzen ist. Eine Grundthese ist dabei, dass Carnaps Werk zwischen den späten 1920ern und den frühen 1930ern dahingehend eine zentrale Kontinuität aufweist, dass die psychophysische Psychologie des späten 19. und frühen 20. Jahrhunderts wichtige Hintergrundannahmen zur Verfügung stellt. Diese These werde ich anhand einer Analyse des Aufbau und der „Psychologie in physikalischer Sprache“ belegen. Zugleich wird jedoch in beiden

U. Feest $(\bowtie)$

Institut für Philosophie, Leibniz Universität Hannover, Hannover, Deutschland

E-Mail: feest@philos.uni-hannover.de

C. Damböck, G. Wolters (eds.), Der junge Carnap in historischem Kontext:

1918-1935 / Young Carnap in an Historical Context: 1918-1935,

Veröffentlichungen des Instituts Wiener Kreis 30,

https://doi.org/10.1007/978-3-030-58251-7_8 
Schriften deutlich, dass Carnaps Bezüge auf die Psychologie bestenfalls eklektisch sind. Beispielsweise beruht seine Behauptung einer Übersetzbarkeit zwischen einer physikalischen und einer eigensychischen Basis auf empirischen Annahmen der Psychologie seiner Zeit, deren Einsichten er an anderer Stelle ignoriert. Insbesondere werde ich zeigen, dass Carnap, obgleich er im Aufbau offiziell noch einer Rekonstruktion der eigenpsychischen Basis verpflichtet ist, Anleihen bei einer Psychologie macht, die grundsätzlich „Fremdpsychisches“ untersucht. In der „Psychologie in physikalischer Sprache“ werden diese Anleihen noch deutlicher, weshalb seine Kritik an der Psychologie bei seinem Diskussionspartner, Karl Duncker, auf Unverständnis stößt. Ob diese Brüche Carnaps philosophischem Projekt schaden, sei dahingestellt. Doch wirft es die grundsätzliche Frage auf, auf welche Weise sich eine wissenschaftliche Philosophie auf die Methoden und Befunde der Einzelwissenschaften beziehen sollte.

Ich beginne in Abschn. 8.2 mit einer kurzen Zusammenfassung der Art und Weise, wie Carnap im Aufbau auf die Psychologie Bezug nimmt, und zeige, warum sein Verweis auf die Gestaltpsychologie in einem gewissen Spannungsverhältnis mit anderen Komponenten seiner Analyse steht. In Abschn. 8.3 beschreibe ich die etwas anders gelagerte Spannung, die wir in Carnaps Schrift zur Psychologie in physikalischer Sprache antreffen und die sich in seinem Schlagabtausch mit dem Psychologen Carl Duncker Ausdruck verschafft. In Abschn. 8.4 schließe ich mit einigen Überlegungen darüber, wie die von mir hervorgehobenen Spannungen einzuschätzen sind.

\subsection{Methodischer Solipsismus und Gestaltpsychologie im Aufbau}

Bei Rudolf Carnaps Der logische Aufbau der Welt (1928) handelt es sich bekanntlich um den Versuch, ein „Konstitutionssystem“ zu erstellen, um zu zeigen, dass sich die Gesamtheit wissenschaftlichen Wissens aus einfacheren Begriffen ableiten lässt. Die von ihm im Aufbau gewählte „Konstitutionsbasis“ ist die „,eigenpsychische“, wobei Carnap jedoch betont, dass seine Entscheidung hierfür eine konventionelle, wenn auch erkenntnistheoretisch begründete, sei. So behauptet er, dass sich eine entsprechende Analyse auch mit einer physikalistischen Basis vollziehen lasse (vgl. $§ 63$ des Aufbau). Damit stellt sich bereits hier die Frage, wie jene beiden Arten möglicher Konstitutionssysteme miteinander kompatibel sind bzw. wie sie sich zueinander verhalten. Ich werde auf diese Frage weiter unten zurückkommen.

Obgleich Carnap noch sein Vorwort zur zweiten Auflage mit der Behauptung beginnt, es gehe ihm um ,die rationale Nachkonstruktion von Begriffen aller Erkenntnisgebiete auf der Grundlage von Begriffen, die sich auf das Gegebene beziehen“"(Carnap 1928/1979, S. X), besagt eine einflussreiche Interpretation dieses Ansatzes, dass es Carnap hier nicht um eine empiristische Fundierung gehe, sondern um die Entwicklung einer neuartigen Konzeption von Objektivität (vgl. Friedman 
1999, S. 129; Uebel 2007, Kap. 1). Diese Objektivität, so weiter, soll dadurch gewährleistet werden, dass die Bedeutung wissenschaftlicher Begriffe durch strukturelle Kennzeichnungen gegeben wird. Diese strukturellen Kennzeichnungen sollen ihrerseits weder einer metaphysischen Struktur der Welt korrespondieren, noch soll dabei auf Anschauungen (Intuitionen) Bezug genommen werden. Vielmehr geht es hier um intersubjektiv geteilte begriffliche Strukturen. Im Zuge der Frage, woraus sich solche intersubjektiv geteilte begriffliche Strukturen speisen, bringt Carnap die Idee einer Basisrelation ins Spiel, der so genannten Ähnlichkeitserinnerung. Diese Wahl mag angesichts des eben Gesagten widersprüchlich anmuten, denn der Begriff der Ähnlichkeitserinnerung deutet auf individuelle subjektive Erfahrungen hin, scheint also im Kontrast zur Forderung einer objektiven Beschreibung begrifflicher Strukturen zu stehen. Carnap spricht dies in $\S 66$ des Aufbau an, wenn er sagt, dass es paradox erscheinen mag, ,,vom individuellen Erlebnisstrom Objektives konstituieren zu wollen“. Er zerstreut die betreffende Sorge jedoch kurz danach, indem er schlicht und einfach konstatiert, dass bestimmte Struktureigenschaften für alle Erlebnisströme übereinstimmen. Deshalb könne man von subjektiven Erfahrungen abstrahieren. Das heißt, Carnap konstatiert, dass sich aus intersubjektiv ähnlich strukturierten Erfahrungen eine objektive Struktur extrahieren lasse. Obgleich klar ist, dass es Carnap letztlich um diese objektive Struktur geht, ist es dennoch bemerkenswert, dass er hier eine empirisch-psychologische Behauptung aufstellt, nämlich dass die Struktureigenschaften unserer Erlebnisströme übereinstimmen. Hier deutet sich schon an, dass Carnap eine psychologische Grundannahme ins Spiel bringt.

Es stellen sich zwei Fragen. Die erste (die in der Literatur einige Aufmerksamkeit erfahren hat) bezieht sich darauf, wie Carnaps erklärtes Ziel einer rein strukturellen Definition wissenschaftlicher Begriffe mit dem Versuch ihrer Fundierung im „Erlebbaren“ zusammenpasst (vgl. z. B. Damböck 2017). So macht Uebel (2007, Kap. 1) beispielsweise eine Grundspannung (,,crucial tension“) im Aufbau aus, die darin besteht, dass Carnap zuletzt weder eine phänomenologische Reduktion noch eine vollständige „Strukturalisierung“ empirischer Prädikate ganz glückt. Solche erkenntnistheoretischen Fragen stehen im vorliegenden Aufsatz jedoch nicht im Vordergrund. Mich interessiert die folgende (zweite) Frage: Carnaps These der intersubjektiven Übereinstimmung der Struktur von Erfahrungsströmen ist in gewisser Weise eine Grundprämisse wahrnehmungspsychologischer Forschung, denn nur unter der Annahme intersubjektiv geteilter Struktureigenschaften von Wahrnehmung kann man in der Forschung Individuen und Stichproben als repräsentativ behandeln. Somit stellt sich die Frage, ob Carnap dabei an irgendwelche Theorien oder Forschungen der wissenschaftlichen oder philosophischen Psychologie seiner Zeit denkt. Einen Hinweis dazu finden wir, zumindest implizit, in $\$ 57$ (,Die physischen Gegenstände sind auf psychische Gegenstände zurückführbar und umgekehrt"). Dort macht er zwei Formen der Rückführbarkeit aus, nämlich erstens die von eigenpsychischen Vorgängen auf (physikalisch beschreibbare) „Parallelvorgänge" im Hirn und zweitens die von fremdpsychischen Vorgängen auf (physikalisch beschreibbare) „Ausdrucksbewegungen“ und „Angaben“. Knapp gesagt deutet Carnap an dieser Stelle also bereits seine Gedanken über die Physikalisierbarkeit der Psychologie an, der dann 1932 weiter ausgeführt wird. In Bezug auf die erste 
These beruft er sich u. a. auf Wilhelm Wundt, erwähnt aber, dass es auch Gegner dieser Auffassung gebe. In Bezug auf die zweite These gibt er keine Referenz. Für meine Zwecke ist wichtig, dass empirische Psychologen wie beispielsweise Wilhelm Wundt natürlich immer mit den Ausdrucksbewegungen und Angaben ihrer Versuchspersonen arbeiten, und zwar unter der Annahme, dass diese intersubjektiv unter ähnlichen Versuchsbedingungen weitgehend übereinstimmen.

Systematisch ist dieser Punkt für mich deshalb zentral, weil er darauf hindeutet, dass Carnap, falls und insofern er sich in seiner Behandlung des Eigenpsychischen auf die Befunde der empirischen Psychologie seiner Zeit beruft, deren Annahmen über die Erforschbarkeit des Fremdpsychischen für seine Rekonstruktion des Eigenpsychischen implizit bereits anerkennen muss. Es liegt daher nahe, dass auch seine Annahme der Strukturgleichheit verschiedener Erlebnisströme aus diesem Kontext stammen. Tatsächlich beruft Carnap sich in seiner Rekonstruktion des Eigenpsychischen im Aufbau zumindest zum Teil auf eine psychologische Strömung seiner Zeit, nämlich die Gestaltpsychologie. Allerdings sind diese Verweise, wie wir gleich sehen werden, mit seinen Aussagen über die Strukturiertheit von Ähnlichkeitserinnerungen nicht konsistent.

In $§ 67$ des Aufbau zitiert Carnap gestaltpsychologische Forschungen (Köhler 1925 und Wertheimer 1925), die (wie der Name schon nahelegt) darauf hinweisen, dass Wahrnehmung ,gestalthaft“, also grundsätzlich strukturiert, sei. Die gestaltpsychologischen Befunde scheinen sich somit hervorragend zu eignen, um Carnaps psychologischer Behauptung eine wissenschaftliche Legitimation zu geben. Wie ich an anderer Stelle (Feest 2007, S. 6-12) gezeigt habe, ist Carnaps Verhältnis zur Gestaltpsychologie aber durchaus gespalten, insofern er einerseits zwar einen wichtigen Aspekt der Theorie der Berlin-Frankfurt-Schule rhetorisch übernimmt, andererseits jedoch eine Grundeinsicht der ganzen gestaltpsychologischen Bewegung in seiner Analyse ignoriert. Diese beiden Punkte sollen zunächst erklärt werden. Fangen wir mit einem kurzen Überblick über die ältere gestaltpsychologische Bewegung an.

Die Tatsache, dass unsere Wahrnehmungen gestalthaft sind, war keineswegs von Köhler und Wertheimer oder den anderen Mitgliedern der so genannten Berlin-Frankfurt-Schule der Gestaltpsychologie entdeckt worden, sondern war bereits spätestens seit den 1890er-Jahren wohlbekannt. Man denke hier beispielsweise an die Tatsache, dass wir drei einzelne Töne bei bestimmten Tonintervallen als einen Dur-Dreiklang wahrnehmen, wohingegen eine leichte Verschiebung der Terz um einen Halbton die Wahrnehmung eines moll-Dreiklangs erzeugt. Innerhalb der Psychologie gab es jahrzehntelang kontroverse Debatten darüber, wie dieses und andere Phänomene zu erklären seien. Christian von Ehrenfels wies darauf hin, dass das Ganze (die Gestalt) mehr als die Summe seiner Teile sei. Damit meinte er, dass beispielsweise ein wahrgenommener Dreiklang einen eigenständigen ontologischen Status gegenüber den Empfindungen der Einzeltöne habe, was man daran erkennen könne, dass man einen Dur-Dreiklang auch in eine andere Tonart transponieren könne, ohne dass er seine Gestalt verlöre. Somit ergab sich die Frage, wie man die Emergenz eines neuartigen Phänomens aus den Einzelteilen erklären könne. Die beiden Hauptantworten verwiesen entweder auf Apperzeption oder Assoziation (vgl. Ash 1995, Kap. 4 und 6). 
Unangetastet blieb durch die genannten beiden Klassen von Erklärungen jedoch eine Grundprämisse, nämlich dass der Wahrnehmung von Gestalten überhaupt einfache Empfindungen vorausgehen. Um dies an einem Beispiel zu verdeutlichen: Ehrenfels behauptete, dass wir beim Hören eines Dreiklanges zunächst einfache Empfindungen der drei einzelnen Töne haben und dann daraus die Wahrnehmung eines Dreiklangs bilden. Die Mitglieder der Berlin-Frankfurt-Schule der Gestaltpsychologie wiesen genau jene zentrale Prämisse der älteren Debatte grundsätzlich von sich, indem sie bestritten, dass wir atomistische Empfindungen als Bausteine von Gestaltwahrnehmungen haben. Damit bestritten sie nicht, dass wir beim Hören eines Dreiklangs zwischen einzelnen Tönen differenzieren können. Sie behaupteten jedoch, dass die betreffenden Wahrnehmungen sekundär gegenüber der Wahrnehmung einer Gestalt seien. Gestalterfahrung ist nach dieser Auffassung eine unmittelbare Wirkung von Stimuluskonfigurationen. Es gibt keine zwischengeschalteten einfachen Empfindungen, die den Elementen der Stimuluskonfigurationen korrespondieren würden. Es dieser Punkt, den auch Carnap aufgreift, wenn er in $\S 67$ des Aufbau schreibt, dass er als Elementarerlebnisse das Gegebene ansehen wolle, also „die Erlebnisinhalte selbst in ihrer Totalität und geschlossenen Einheit“. Aus dieser geschlossenen Einheit könne man dann atomistische Empfindungen höchstens per Abstraktion gewinnen, doch seien diese Empfindungen keine ontologisch primäre Bestandteile der Gestaltwahrnehmungen. Aus diesem Grund spricht er in diesem Zusammenhang auch von „Quasizerlegung“, der höchstens die Identifikation von Quasi-Bestandeilen gelingen könne (§ 71).

Nun könnte man auf die Idee kommen, dass Carnaps Begriff der Ähnlichkeitserinnerung, der - wie gesagt - fundamental für seine Analyse begrifflicher Strukturen ist, direkt an die Befunde der Gestaltpsychologie anknüpft. Denn bereits Ehrenfels' Begriff der Gestalt hatte ja nahegelegt, dass die Ähnlichkeit zwischen zwei Wahrnehmungen (etwa der Wahrnehmung zweier moll-Dreiklänge in verschiedenen Tonarten) auf der Gestalt des moll-Dreiklanges beruht und eben nicht auf Ähnlichkeiten zwischen den Stimuluselementen. Paradoxerweise geht aber Carnap diesen Weg nicht, sondern bestimmt stattdessen Ähnlichkeitserinnerung durch die Gemeinsamkeit von Quasielementen (also der Empfindungen einzelner Töne, die in den Dreiklängen vorkommen). Durch seine Wahl des Wortes „Quasielement“ gibt Carnap zu erkennen, dass er die Einsicht der Berlin-Frankfurt-Schule der Gestaltpsychologie verinnerlicht hat, der zufolge die Empfindungen komplexer Tonempfindungen sich eben nicht aus einfachen Tonempfindungen zusammensetzen. Als Resultat dessen, dass er sich bei der Analyse von Ähnlichkeitserinnerungen dennoch auf die (fiktiven) Quasiempfindungen beruft, folgt, dass die von ihm identifizierten Strukturen der Ähnlichkeitserinnerung radikal von denen abweichen, die man aufgrund der gestaltpsychologischen Theorie erwarten würde: Ausschlaggebend für eine Ähnlichkeitserinnerung ist grade nicht die relative Stimuluskonfiguration, sondern lediglich das Vorliegen gemeinsamer Quasi-Elemente. Dies wird bereits in Carnaps Manuskript „Die Quasizerlegung“ (1923) ausgeführt, wobei herauskommt, dass nach der gestaltpsychologischen und nach der Carnapschen Analyse jeweils ganz unterschiedliche Akkorde als ähnlich klassifiziert würden (vgl. Feest 2007, S. 11). 
Zusammenfassend lässt sich also konstatieren, dass Carnaps Bezugnahme auf die Gestaltpsychologie im Aufbau insofern höchst selektiv ist, als er zwar von Mitgliedern der Berlin-Frankfurt-Schule die Idee des Primats der Gestalt übernimmt, sich jedoch nicht dafür interessiert, wie die (empirisch eruierten) Ähnlichkeitsrelationen zwischen Gestalten innerhalb dieser Tradition gedacht werden. Um es noch schärfer auszudrücken: Seine Rekonstruktion der Ähnlichkeitserinnerung darf als empirisch falsch gelten, zumindest wenn man die experimentellen Ergebnisse der in der psychophysischen Tradition arbeitenden Gestaltpsychologen zugrunde legt. Was geht hier also vor? Eine mögliche plausible Lesart ist, dass die tatsächliche Psychologie des Erkenntnisprozesses für Carnap schlicht irrelevant ist, da es ihm eher um eine logisch-rationale Rekonstruktion geht. ${ }^{1}$ In der neueren Literatur gibt es jedoch noch eine andere Interpretation, der zufolge sich zumindest der junge Carnap sehr wohl für tatsächliche Erkenntnisprozesse interessierte, diese jedoch nicht im Sinne der experimentellen Psychologie seiner Zeit konzeptualisierte. In diesem Sinne argumentiert etwa Christian Damböck, dass Carnap in eine ,empirische“, aber nicht experimentelle Tradition der deutschsprachigen Philosophie und Psychologie einzuordnen sei (Damböck 2017, Kap. 4).

Carnap wählt zwar im Aufbau eine „eigenpsychologische Basis“ für sein Konstitutionssystem, betont jedoch schon zu diesem Zeitpunkt die Übersetzbarkeit (,Überführbarkeit“") der eigenpsychologischen in eine physikalische Sprache. Ein Argument hierfür liefert die empirische Behauptung (in $§ 57$ ) des psychophysischen Parallelismus. Interessanterweise wird an dieser Stelle auch die Möglichkeit einer „fremdpsychischen“ Fundierung angesprochen (die ihrerseits in die physikalische Beschreibung von Verhalten überführbar sei). In $§ 58$ wägt Carnap die Frage nach einer eigen- vs. fremdpsychischen Fundierung ab, spricht sich dann jedoch für die eigenpsychische Fundierung aus, denn fremdpsychische Gegenstände seien nur auf dem Umweg über Verhaltensbeobachtungen möglich. „,Dagegen bedarf die Erkennung der eigenen psychischen Vorgänge nicht irgendwelcher Vermittlung durch die Erkennung physischer Gegenstände, sondern geschieht unvermittelt" (Carnap 1979/1928, S. 57). Aus diesem Grunde komme das Fremdpsychische in der ,erkenntnistheoretischen Primarität" (ebd.) auch erst nach dem Eigenpsychischen und dem Physischen. Wie ich gerade argumentiert habe, besteht die Methode der wissenschaftlichen Psychologie jedoch entscheidend auf der Erforschung des Fremdpsychischen: Mentale Zustände werden auf dem Wege der Verhaltensbeobachtung (inklusive der Beobachtung verbaler Kundgaben) beschrieben. Aus $§ 57$ geht hervor, dass Carnap dies bekannt war. Wenn er sich in seinen Ausführungen über die Strukturiertheit auf die Befunde der Gestaltpsychologie beruft, setzt er somit Befunde voraus, die sich „fremdpsychologischer“ Methoden bedienen. Zugleich ignoriert er aber deren tatsächliche Befunde zugunsten seiner ,eigenpsychologischen“ Analyse. Anders ausgedrückt präferiert Carnap hier also letztlich eine Methode, die man heute vermutlich als „Lehnstuhlmethode“ bezeichnen würde.

\footnotetext{
${ }^{1}$ Ich bedanke mich bei Thomas Uebel, der diese Interpretation in seinen Kommentaren zu einer früheren Version des vorliegenden Artikels stark gemacht hat.
} 


\subsection{Die Physikalisierung der psychologischen Sprache}

Aus dem bisher Gesagten geht hervor, dass Carnaps Konzeptualisierung des Eigenpsychischen im Aufbau einer gewissen Spannung unterliegt: Einerseits wird es als „erkenntnismäßig primär" und damit außerhalb des physikalischen und fremdpsychologischen Zugangs gesetzt. Andererseits behauptet er bereits hier, dass das Eigenpsychische grundsätzlich physikalisierbar sei und außerdem eine intersubjektiv geteilte Struktur aufweise. Die letzteren beiden Behauptungen, so scheint mir, werden erst mit Carnaps Hinwendung zum Physikalismus eingeholt. In gewisser Weise wird erst mit dem Physikalismus deutlich, wie ein physikalistisches und ein eigenpsychologisches Konstitutionssystem überhaupt kompatibel sein können. Für Carnap wird eine solche Kompatibilität dadurch möglich, dass sich - wie bereits in $\S 57$ des Aufbau angedeutet - die Sätze der einen Sprache in solche der anderen übersetzen lassen. Um argumentieren zu können, dass eine Übersetzbarkeit in der Tat gegeben ist, so meine These in diesem Abschnitt, muss Carnap aber auch hier wieder Anleihen bei der seinerzeit bestehenden Psychologie machen. Dabei wird jedoch deutlich, dass auch die 1932 existierende Psychologie noch keine Übersetzung von einem psychologischen in ein hirnphysiologisches Vokabular zulässt. Carnap konzentriert sich daher auf die Übersetzbarkeit in ein Verhaltensvokabular, also in ein fremdpsychologisches Vokabular. ${ }^{2}$ Insofern finden wir eine direkte Kontinuität zwischen $§ 57$ des Aufbau und dem Projekt der Physikalisierung der psychologischen Sprache. Sein Bezug auf die Psychologie, so argumentiere ich, ist in gewisser Weise konsistenter als im Aufbau (er formuliert im Prinzip die von der empirischen Psychologie praktizierte fremdpsychologische Methode), doch distanziert er sich in diesem Text deutlich von der empirischen Psychologie, mit dem Argument, dass diese nach wie vor einer nicht-physikalistischen Methode verpflichtet sei. Im Folgenden soll Carnaps Gedankengang bezüglich einer universalen physikalischen Sprache expliziert werden.

In seinem Artikel „Überwindung der Metaphysik durch logische Analyse der Sprache“ (Carnap 1931a) legt Carnap seine verifikationistische Semantik vor, der zufolge ein „Wort“ (Prädikat) $P$ nur dann Bedeutung hat, wenn (i) empirische Anwendungsbedingungen von $P$ bekannt sind, (ii) bekannt ist, aus welchen Protokollsätzen sich ein Elementarsatz $S(a)$, in dem $P$ als Prädikat fungiert, ableiten lässt, und (iii) bekannt ist, wie man $S(a)$ empirisch prüfen kann. Damit erhebt sich aber die Frage, woraus die Protokollsätze selbst ihre Bedeutung beziehen. Hier handelt es sich natürlich abermals um die Frage nach dem Status der Sätze, die empirische Elementarerlebnisse beschreiben, also genau der Art von Sätzen, die Carnap im Aufbau zur Grundlage seines Konstitutionssystems erhoben hatte. Allerdings geht es hier nicht darum, eine Aussage über die Struktur solcher Elementarerlebnisse zu treffen, sondern darum, Kriterien für die Bedeutungshaltigkeit der Erlebnissprache anzugeben.

\footnotetext{
${ }^{2}$ Ich möchte betonen, dass Carnap hier die Idee der Physikalisierung des Eigenpsychischen keineswegs hinter sich lässt, sondern lediglich bemerkt, dass eine solche beim Stand der Forschung noch nicht möglich sei. Zugleich scheint er im Verlaufe des Textes einzuräumen, dass auch eine solche Physikalisierung den Weg über das Fremdpsychische gehen muss.
} 
Auf diese Frage antwortet Carnap in seinem Aufsatz „Über die physikalische Sprache als Universalsprache der Wissenschaften“ (Carnap 1932a), indem er argumentiert, dass alle bedeutungsvollen Sätze (also inklusive der Sätze der Protokollsprache) in eine universelle wissenschaftliche Sprache zu übersetzen seien, wobei er mit „Universalität“ meint, dass eine solche Sprache alle Sachverhalte beschreiben könne. Außerdem sollte diese Sprache intersubjektiv sein. Carnap betrachtet diese These von der physikalischen Sprache als Universalsprache für alle wissenschaftlichen Disziplinen zumindest für die Biologie als intuitiv einleuchtend. Im Falle der Physikalisierbarkeit von Protokollsätzen führt er aus, dass diejenigen Sätze, die im Aufbau unter Rekurs auf eine eigenpsychische Basis fundiert worden waren, sich in Sätze der ,physikalischen Sprache“ übersetzen lassen. Im materialen Modus ausgedrückt laufe dies darauf hinaus, dass es funktionale oder gesetzmäßige Beziehungen zwischen spezifischen physischen Zuständen und Wahrnehmungen gebe. Nun ist natürlich klar, dass es Carnap darum geht zu argumentieren, dass ein Verharren im materialen Modus zu philosophischen Scheinproblemen führen kann, die durch seine metaphilosophischen Überlegungen gerade überwunden werden sollen. Insofern sollten Carnaps Ausführungen auch nicht primär als an die Psychologie gerichtet gelesen werden. Dennoch weist, wie wir gleich sehen werden, seine Beschreibung der Physikalisierung bemerkenswerte Parallelen zu den tatsächlichen Methoden der experimentellen Psychophysik seiner Zeit auf.

Obgleich Carnap primär an das Verhältnis zwischen Sätzen der Hirnphysiologie und der Psychologie denkt, ist ihm natürlich bewusst, dass solche funktionalen Abhängigkeiten beim damaligen Stand der Hirnphysiologie (noch) nicht formulierbar sind. Dasselbe konstatiert er bereits im Aufbau (\$ 57), doch dient es ihm dort letztlich als (etwas zweifelhaftes) Argument für die Direktheit des eigenpsychologischen Zuganges, also gegen eine Methode, die den Umweg über physikalistische Verhaltensbeschreibungen geht. In seiner „Psychologie in physikalischer Sprache“ vollzieht er an genau diesem Punkt eine Kehrtwende und entscheidet sich (wenn auch in etwas anderer Terminologie) für eine fremdpsychologische Rekonstruktion des psychologischen Vokabulars. Als relevante physikalistische Beschreibungen lässt er daher Sätze über Verhaltensdispositionen gelten, die durch bestimmte physikalische Stimulus-Konfigurationen aktualisiert würden. Konkret geht es um die Disposition, als Reaktion auf bestimmte physische Stimuli mit bestimmten Protokollsätzen zu reagieren. So ließen sich etwa gesetzmäßige Zusammenhänge über die funktionale Abhängigkeit zwischen der Beschreibung physischer Stimuli und Verhaltensweisen formulieren, die es ihrerseits erlauben würden, Aussagen über Erfahrungen als extensionsgleich mit Aussagen über Verhaltensdispositionen zu behandeln.

In diesem Sinne beschreibt Carnap eine Art Versuchsanordnung, mit deren Hilfe man die genannten funktionalen Abhängigkeiten erforschen könne: „Das Verfahren besteht darin, daß $S_{1}$ die physikalischen Bedingungen (etwa die Kombination verschiedener Schwingungsfrequenzen) variiert und feststellt, unter welchen Bedingungen $S_{i}$ mit einem Protokollsatz reagiert, der die betreffende qualitative Bestimmung enthält" (Carnap 1931b, S. 446). Er fährt dann fort, dass er die Entdeckung solcher physikalischen Bestimmungen, die bestimmten qualitativen Bestimmungen 
zugeordnet werden können, als „Physikalisierung“ bezeichnen wolle (ebd.). Die Tatsache, dass dies möglich sei, sei einem ,glücklichen Umstand zu verdanken“, nämlich dem ,allgemein ordnungshaften Zug der Erfahrung“ (Carnap 1931b, S. 447).

Obgleich Carnap dies nicht erwähnt, ist für Psychologiehistoriker*innen unmittelbar ersichtlich, dass das von Carnap hier beschriebene Forschungsprogramm der Variierung physikalischer Bedingungen mit dem Ziel der Erstellung gesetzmäßiger Beziehungen zwischen Stimuli und diskriminativem Verhalten (verbaler oder nonverbaler Art) sich spätestens seit Gustav Theodor Fechner 1860 wird hier zitiert Werk Elemente der Psychophysik (1860) einer großen Beliebtheit erfreute, wenngleich es natürlich Fechner und anderen Psychophysikern nicht darum ging, eine universelle physikalische Sprache zu entwickeln, sondern vielmehr (im „materialen Modus“ sozusagen) schlicht und einfach psychophysische Zusammenhänge zu erforschen. Dennoch ist es wichtig hervorzuheben, dass die experimentellen Paradigmen der psychophysischen Forschung (inklusive derjenigen der Gestaltpsychologen, auf welche Carnap sich bereits im Aufbau bezogen hatte) exakt nach dem Vorbild von Carnaps fiktiver Versuchsanordnung vorgehen. Weiterhin möchte ich darauf hinweisen, dass ein solches psychophysikalisches Forschungsparadigma auch von den Gestaltpsychologen praktiziert wird, auf die Carnap sich schon 1928 im Aufbau bezieht.

Genau dies wird, wie ich gleich näher ausführen werde, auch von Karl Duncker, einem jüngeren Vertreter der Gestaltpsychologie, bemerkt (so behaupte ich jedenfalls), als er 1932 mit Unverständnis (Duncker 1932) auf Carnaps Artikel über die Psychologie in physikalischer Sprache reagiert. Dieses Unverständnis ist durchaus nachzuvollziehen, denn Carnap suggeriert, trotz seines primär metaphilosophischen Anspruches, dass die experimentelle Psychologie selbst wohl beraten sei, sich seine Analyse zu Herzen zu nehmen. So schreibt er beispielsweise in seinem Artikel „Psychologie in physikalischer Sprache“: „An den Psychologen sind hiernach zwei Forderungen zu stellen. Erstens muss er sich [...] darüber klar werden, dass er [...] nichts anderes feststellt, als das Vorliegen eines bestimmten physikalischen Zustandes der Versuchsperson [...]. Zweitens muss er [...] die Physikalisierung [...] als Aufgabe der wissenschaftlichen Forschung anerkennen“ (Carnap 1932a, S. 129). Konkret macht Carnap zwei Klassen von problematischen psychologischen Beobachtungssätzen aus, nämlich einerseits Sätze über das Fremdpsychische und andererseits Selbstbeobachtungen. In Bezug auf das Problem des Fremdpsychischen argumentiert er etwa, dass seine Analyse impliziere, dass man nur dann bestimmte mentale Zustände zuschreiben dürfe, wenn man das Verhalten des betreffenden Menschen unter einen allgemeinen Satz subsumieren könne, der eine regelmäßige Beziehung zwischen bestimmten Stimulusbedingungen und bestimmten Verhaltensweisen beschreibt. Dies kontrastiert er mit der (von ihm abgelehnten) Methode, bei der die Zuschreibung von Erlebniszuständen eher intuitiv vorgenommen werde. In Bezug auf das Problem der Introspektion argumentiert Carnap ganz parallel, indem er sagt, dass ,introspektive“ Sätze wie „Ich bin gerade nervös“ entweder bedeutungslos seien oder sich empirische Wahrheitsbedingungen für sie angeben lassen. Er erklärt außerdem, dass es ihm um ein Analysewerkzeug zur Vermeidung metaphysischen Unsinns gehe. Solch metaphysischer Unsinn entstehe beispiels- 
weise, wenn man von subjektiven mentalen Zuständen als Gegenständen introspektiver Forschung spreche, als ob diese irgendwie zusätzlich zu den relevanten physikalischen Gegebenheiten existierten. Zusätzlich warnt er davor, introspektiv zugänglichen Erfahrungen einen besonderen epistemischen Status zuzubilligen, und setzt dagegen, dass man es immer mit Beobachtungssätzen (also mit öffentlich zugänglichen physikalischen Daten) zu tun habe.

Ich möchte noch einmal betonen, dass Carnaps Projekt ein metaphilosophisches ist, insofern es ihm um die Reinigung der psychologischen Sprache von Elementen geht, die zu metaphysischen Missverständnissen führen könnten. Er gesteht daher auch zu, dass die Psychologen in ihrer Forschungspraxis natürlich weiterhin ,,verstehende“" und ,introspektive“ Sätze verwenden dürften, solange sich diese physikalisieren ließen. Dennoch bleibt auffällig, dass er „der Psychologie“ sehr pauschal unterstellt, dass sie dies nicht immer angemessen tue. Nun stellt sich die Frage, welche zeitgenössischen Psychologen er hier genau im Auge hat, die vermeintlich die von ihm angeprangerten Fehler begehen. An diesem Punkt ist Carnap leider nicht sehr konkret. Es gibt allerdings Hinweise darauf, dass ein unmittelbares Ziel seiner Kritik der Psychologe Karl Bühler war, der 1926 einen Artikel mit dem Titel „Die Krise der Psychologie“ veröffentlicht hatte, gefolgt von einem Buch gleichen Namens (Bühler 1926, 1927). In diesen Werken hatte Bühler den „Physikalismus“ als Allheilmittel gegen die methodische und theoretische Fragmentierung der Psychologie kritisiert. Für diese These spricht auch, dass Carnap am 28. Mai 1930 einen Vortrag in Bühlers psychologischem Kolloquium hielt. ${ }^{3}$ Aufgrund von Carnaps Vortragsmanuskript wird ersichtlich, dass der Inhalt des Vortrags den 1932 veröffentlichten Artikeln bereits recht nahe kommt. Es steht zu vermuten, dass die in Bühlers Kolloquium geführte Diskussion in Carnap (1932a) ihren Niederschlag findet. Dabei ist es gut denkbar, dass es Carnap nicht primär um eine Kritik Bühlers geht, sondern vielmehr um eine Klärung des Begriffes der Physikalisierung. Zu spekulieren ist außerdem, dass Carnap bei seiner Kritik des Verstehens (abermals als intuitives Einfühlen verstanden) an Wilhelm Dilthey denkt. Für diese Lesart könnte angeführt werden, dass Carnap nach der Analyse von Christian Damböck (2017) in den frühen 1920er-Jahren (bis hin zu frühen Manuskripten des Aufbau) noch Anleihen bei Diltheys deskriptiver Psychologie macht (vgl. Damböck 2017, Kap. 4). Es ist daher nicht unplausibel, dass Carnap hier nicht zuletzt eine bestimmte Psychologiekonzeption ablehnen möchte, die für ihn biographisch relevant war, die aber eine große Entfernung zu experimentalpsychologischen Arbeiten aufweist. Somit kann spekuliert werden, dass sich Carnap mit seiner Kritik der Psychologie von den nicht-physikalistisch gefassten Anteilen seiner früheren Konzeption des „Eigenpsychischen“ distanziert. Die Namen Bühler und Dilthey werden jedoch von Carnap nicht explizit genannt.

Kommen wir nun also zu seiner Auseinandersetzung mit Duncker. Wie bereits erwähnt, weist die von Carnap vorgeschlagene Idee der Physikalisierung der Psy-

${ }^{3}$ Lectures in Europe (Items 30-42), 1929-1933. Box 110b, Folder 7c. Rudolf Carnap Papers, 1905-1970, ASP.1974.01, Special Collections Department, University of Pittsburgh. 
chologie eine große Ähnlichkeit zu den Methoden der Psychophysik auf. Carnap stellt diese Verbindung aber nicht her, sondern verweist stattdessen auf den Behaviorismus von J. B. Watson. Das passt jedoch wenig zu seinem Anliegen, denn der Behaviorismus hatte ja die gänzliche Eliminierung mentalistischer Begriffe gefordert, während es Carnap darum geht, die Bedingungen zu explizieren, unter denen mentalistische Wörter und Sätze bedeutungsvoll sein können. Carnaps Vorgehen ist also dem der Identitätstheorie des Geistes ähnlich, die ja nicht sagt, dass subjektive mentale Zustände nicht existieren, sondern lediglich zu explizieren sucht, was sie genau sind (in Klammern sei kurz hinzugefügt, dass - wie Michael Heidelberger (2003) gezeigt hat - solche identitätstheoretischen Ansätze in der Tradition der Psychophysik bereits in der Mitte des 19. Jahrhunderts formuliert worden waren, wenngleich Fechner, etwas unglücklich, den Begriff „Parallelismus“ verwendet hatte). Entsprechend zielt Carnap hier nicht darauf ab zu sagen, dass subjektive Terme bedeutungslos sind, sondern er führt vielmehr Kriterien der Bestimmung ihrer Bedeutungshaftigkeit ein.

Diesen letzten Punkt (also Carnaps Projekt der formalen Analyse von Sprache) versteht Duncker offenkundig nicht, sondern er hakt stattdessen bei Carnaps Erklärungen im materialen Modus nach, bei denen Carnap ja quasi unter Verweis auf eine Art fiktiven psychophysischen Gedankenexperimentes die Übersetzbarkeit psychologischer in physikalische Sätze illustriert und begründet hatte. Dementsprechend weist Duncker zu Recht darauf hin, dass die Psychophysik dem Carnapschen Projekt viel näher stehe als der Behaviorismus. Duncker bemerkt außerdem, dass ihm (zumindest unter modernen wissenschaftlich gesinnten Psychologen) niemand bekannt sei, der die von Carnap benannten Fehler tatsächlich begehe (zur Erinnerung: Bei diesen Fehlern handelt es sich um den der ontologische Doppelung sowie um die Annahme, Introspektion könne eine epistemisch privilegierte Art von Daten liefern). Insbesondere betont er die physikalistische und antimetaphysische Ausrichtung der Gestaltpsychologie. Duncker sagt daher, dass er nicht wisse, wen Carnap überhaupt meine.

Obgleich natürlich zugestanden werden muss, dass Duncker die metaphilosophische Stoßrichtung von Carnaps Argument nicht versteht, halte ich Dunckers Befremden über das völlige Ignorieren der Psychophysik in Carnaps Artikel dennoch für nachvollziehbar: Psychophysikalische Forschung untersucht eben genau die funktionalen Abhängigkeiten zwischen Umweltreizen und Protokollsätzen, die für eine Physikalisierung der Protokollsätze benötigt würden, wenn man diese tatsächlich durchführen wollte. Leider ist Duncker aber terminologisch nicht besonders vorsichtig und so schleichen sich in seine Antwort auf Carnap einige Formulierungen ein, die so klingen, als ob er doch eben die Fehler beginge vor denen Carnap warnen möchte. Dementsprechend ist es für Carnap ein Leichtes, Duncker verworrener Formulierungen zu überführen. So sagt er in großer Schärfe, dass Duncker ihn offenbar überhaupt nicht verstanden habe und außerdem ein völlig anderes Verständnis von Physikalismus besitze als er selbst (Carnap 1932b). Ihm, Carnap, gehe es um die Übersetzbarkeit einer psychologischen in eine physische Sprache, während es Duncker offenbar um die Identifikation einer physischen Basis für introspektiv zugängliche subjektive Zustände gehe. Dabei ignoriert Carnap unter anderem, dass sich 
Duncker ausdrücklich von der Annahme distanziert, dass es sich bei solchen mentalen Zuständen um irreduzible Qualia handele. Er ignoriert außerdem, dass das von ihm propagierte Programm der Übersetzbarkeit physikalischer und ,psychologischer" Begriff eben jene (materialen) Voraussetzungen benötigt, die auch von der psychophysischen Forschung gemacht werden.

Zusammenfassend lässt sich also konstatieren, dass Carnap und Duncker in ihrem Austausch von 1932 aneinander vorbeireden. Somit können beide nicht herausarbeiten, dass das psychophysikalische Paradigma der Psychologie (in dessen Tradition sich der Gestaltpsychologe Duncker selbst verortet) genau die Art von Forschung betreibt, die Carnap (in fiktiver Form) seinem Programm der Physikalisierung er ,psychologischen“ Sprache zugrunde legt.

\subsection{Mögliche Stränge einer Bewertung der Debatte}

Von außen und mit etwas historischer Distanz betrachtet, scheint es, dass die Missverständnisse relativ leicht hätten behoben werden können und dass vor allem Carnap hier eine Chance für einen genuinen Dialog verstreichen lässt (vgl. auch Feest 2017, S. 123). Das deutet darauf hin, dass Carnap sich für die real praktizierte experimentelle Psychologie eigentlich nicht interessierte, da sie für sein eigenes philosophisches Projekt recht irrelevant war.

In diesem Punkt bleibt der Carnap von 1932 dem Carnap von 1928 treu, wenn auch aus geänderten Gründen. Dort hatte er sich in seiner Rekonstruktion der eigenpsychologischen Basis ja explizit auf eine Grundannahme der Berlin-FrankfurtSchule der Gestaltpsychologie (die Strukturiertheit einfacher Empfindungen) bezogen, ohne zu berücksichtigen, dass die Gestaltpsychologie als psychophysikalisch vorgehende Wahrnehmungsforschung einer fremdpsychologischen Methode verpflichtet war. Carnaps Ansatz in den frühen 1930er-Jahren hingegen, mit der These einer Übersetzbarkeit psychologischer und physikalischer Begriffe, setzt hingegen in gewisser Weise selber auf eine fremdpsychologische Methode (auch wenn diese von ihm nicht experimentell eingesetzt wird), ohne anzuerkennen, dass eine solche Methode in der psychophysischen Forschung bereits gang und gäbe ist.

Gegen meine kritische Beurteilung der Tatsache, dass für Carnap die real praktizierte Psychologie und ihre Geschichte eher uninteressant waren, kann eingewandt werden, dass die im vorliegenden Aufsatz geleistete Analyse für eine Bewertung des systematischen Projekts Carnaps belanglos ist. In diesem Sinne könnte ein Kritiker meiner Analyse beispielsweise darauf hinweisen, dass Carnaps selektive und inkonsistente Bezugnahme auf die Psychologie im Aufbau seinem erkenntnistheoretischen Ziel nicht schadet, da es ja explizit um rationale Rekonstruktion subjektiver und psychologischer Erkenntnisansprüche ging und nicht um realistische Beschreibung des Erkenntnis- oder Forschungsprozesses. Der Einwand kann als berechtigt angesehen werden, wirft aber grundsätzliche, und bis heute relevante, Fragen darüber auf, wie eine angemessene philosophische Bezugnahme auf psychologische Forschung aussehen kann. Gegen den Einspruch, dass eine inkonsistente und eklek- 
tische Bezugnahme auf die Psychologie das Carnapsche Projekt selbst nicht automatisch inkonsistent macht, möchte ich dennoch zu bedenken geben, dass eine konsistente Bezugnahme, oder zumindest eine explizite Reflexion auf Ähnlichkeiten und Unterschiede zwischen der real existierenden Psychologie und der Carnapschen Verwendung der betreffenden Forschung, eleganter wäre. In diesem Sinne erscheint es mir als problematisch, im Zuge des Projekts einer wissenschaftlichen Philosophie nur ausgewählte Aspekte des Korpus einer Wissenschaft zu berücksichtigen.

Abgesehen von der Frage, wie Carnaps selektive Bezugnahme auf die Psychologie normativ zu beurteilen ist, habe ich jedoch auch eine historische Behauptung aufgestellt, nämlich dass Carnaps scheinbare Anleihen bei der psychophysischen Tradition der experimentellen Psychologie nicht zufällig sind, sondern dass ihm die betreffenden Denkfiguren und Forschungspraktiken tatsächlich bekannt waren, obgleich ihm das tatsächliche begriffliche Verhältnis beispielsweise zwischen Psychophysik und Behaviorismus offenbar nicht ganz klar wird. Es könnte kritisch bemerkt werden, dass ich konkrete historische Belege für diese These schuldig geblieben bin. Auch dieser Kritikpunkt ist berechtigt, doch scheint mir die Annahme, dass Carnap zumindest eine oberflächliche Vertrautheit mit der Psychologie hatte, dennoch plausibel. Damit sei nicht in Abrede gestellt, dass es auch andere wichtige Einflüsse gab, die eher aus der nicht-experimentellen philosophischen Psychologie kamen, die das Projekt der Bewusstseinsanalyse seit dem 19. Jahrhundert in den Dienst der Metaphysikkritik stellte (vgl. Feest 2007, Abschn. 5).

Im Zusammenhang der Frage nach solchen anderen Einflüssen möchte ich noch einmal auf Carnaps Begriff der eigenpsychologischen Basis zurückkommen, den er (ohne dies näher zu erläutern) mit der Idee des methodologischen Solipsismus gleichsetzt. Wie bereits weiter oben ausgeführt, verwendet er den Begriff der eigenpsychologischen Basis zweideutig, indem er einerseits nahelegt, dass eine Analyse auf eigenpsychologischer Basis jeglicher physikalischer Methode vorangeht, andererseits aber die empirische These aufstellt, dass sich das auf dieser Basis konstruierte Vokabular in ein physikalisches übersetzen lasse. Ich möchte (vorsichtig) argumentieren, dass wir im Aufbau eine gewisse Unentschiedenheit zwischen zwei verschiedenen antimetaphysischen Strategien sehen. Die eine Strategie ist ,vorwärtsgewandt" und nimmt bereits die spätere antimetaphysische Strategie der Übersetzung aller Sätze in eine physikalische Sprache vorweg. Der Grundgedanke hierbei ist, dass metaphysische Spekulationen durch radikale Konzentration auf Sprachanalyse vermieden werden können und dass die Übersetzbarkeit aller Vokabulare in ein physikalisches empirisch eingeholt werden kann.

Die zweite Strategie ist ,rückwärtsgewandt“ und knüpft eher an die Methode der radikalen Bewusstseinsanalyse an, die den metaphysischen Status der Außenwelt explizit ausklammert. Eine solche Strategie finden wir beispielsweise bei Edmund Husserl, der in diesem Zusammenhang ebenfalls den Begriff des methodischen Solipsismus verwandte. Husserl meinte damit eine Methode der radikalen Enthaltsamkeit gegenüber der Frage, wie sich Phänomene des Bewusstseins auf die Phänomene der materiellen Welt beziehen. Die Methode der Phänomenologie besteht dementsprechend in einer Urteilsenthaltung (Epoché) und einer Konzentration auf Bewusstseinsphänomene mit dem Ziel der Wesensschau (vgl. z. B. Husserl 
1913/2009). ${ }^{4}$ Es ist klar, dass Carnap mit dem Begriff des methodischen Solipsismus nicht Husserls Projekt verbindet. Dennoch gibt es ein gemeinsames Motiv, nämlich das der ontologischen Enthaltsamkeit, also einer Einstellung, die aus methodischen Gründen ganz bewusst auf ontologische Positionierungen verzichtet. In diesem Sinne weist etwa der Husserl-Interpret Zahavi (2011) darauf hin, dass es Husserl darum gegangen sei, ,eine gewisse dogmatische Einstellung gegenüber der Wirklichkeit außer Kraft zu setzen oder zu neutralisieren" (Zahavi 2011, S. 106). Der Solipsismus ist also deshalb ein methodischer, weil er (entsprechend dem methodischen Zweifel bei Descartes) nur als philosophisches Werkzeug verstanden wird. In diesem Sinne scheint es mir sinnvoll, auch Carnaps methodischen Solipsismus im Aufbau als anti-dogmatistisches Werkzeug zu deuten, ein Werkzeug, das in gewisser Weise genau das mit bezwecken soll, was Carus als die „Flucht vor der Ontologie" bezeichnet (Carus 2016, S. 141). Carnap möchte (wie Husserl) über eine Subjekt-Objekt-Trennung hinaus, insofern er letztlich eine vollständige Strukturbeschreibung eines Konstitutionssystems anstrebt, die nicht zwischen Innen und Außen unterscheidet.

Allerdings ist unklar, ob das fragliche Werkzeug die antimetaphysische Flucht vor der Ontologie tatsächlich ermöglichen kann, denn zumindest eine Klasse von Gegenständen, die Basisrelationen, müssen ja nach wie vor gesetzt werden, sie scheinen also durch Carnaps methodischen Solipsismus intakt gelassen zu werden. Hier sind wir wieder mit dem Aspekt des Aufbau konfrontiert, den Uebel (2007, S. 57) als „crucial tension“ bezeichnet hat, einer Spannung, von der Carus (2016) argumentiert, dass sie aus einer ,naturalistischen Zwickmühle“ entstehe, in der wir uns finden, wenn wir menschliche Erfahrung gleichzeitig als Erkenntnisgrundlage und als natürlichen Gegenstand behandeln wollen. Carnaps versuchter Ausweg aus der Zwickmühle besteht darin, Erfahrungssätze in Struktursätze auflösen zu wollen, doch ,though Ursprünglichkeit and Struktur are mostly not in open or direct conflict [in the Aufbau] (apart from the notorious $\S \S 153-5$ ), the tension simmers under the surface" (Carus 2016, S. 147).

Carnaps methodischer Solipsismus ist also (insofern es um eine Suspendierung dogmatischer Einstellungen geht) Ausdruck seines antimetaphysischen Grundimpulses. Zugleich treten im Aufbau aber die Grenzen einer solchen Suspendierung von Einstellungen zutage, nämlich wenn es um die phänomenale Grundlage der Einstellungen geht. Dies wird deutlich an den Schwierigkeiten einer konsequenten „Strukturalisierung“ der eigenpsychologischen Basis des methodischen Solipsismus. Ohne dies hier ausführen zu können, behaupte ich, dass sich hier der zentrale Punkt findet, der Carnap dazu beweget, im weiteren Verlauf eine im Aufbau ebenfalls bereits angelegte zweite antimetaphysische Strategie zu verfolgen: die der Forderung der Übersetzbarkeit aller sinnvollen Sätze in eine physikalistische Universalsprache. In gewisser Weise handelt es sich hier um einen anderen Modus der

\footnotetext{
${ }^{4}$ Auf ähnliche Weise, wenngleich in einem ganz anderen Kontext, verwendet auch der Philosoph Jerry Fodor den Ausdruck ,methodischer Solipsismus“ und meint damit eine Konzentration auf kognitive Prozesse, gepaart mit einem radikalen Agnostizismus gegenüber deren semantischem Gehalt.
} 
Suspendierung dogmatischer Einstellungen, einen Modus, der die physikalistische Sprachanalyse gegenüber der solipsistischen Bewusstseinsanalyse betont und damit versucht, die im Aufbau angelegte zentrale Spannung zu überwinden. Während also der methodische Solipsismus sich um eine strukturelle Beschreibung von Erfahrung bemühte, geht die physikalistische Sprachanalyse so vor, dass sie nach formalen Kriterien der Bedeutungshaltigkeit aller Sätze fragt, und zwar inklusive derjenigen Sätze, die Erfahrungstatsachen ausdrücken. Vor diesem Hintergrund wird vielleicht Carnaps Irritation darüber verständlich, dass Duncker diesen für Carnap so zentralen Punkt nicht sieht.

\section{Schluss}

Der vorliegende Artikel hat versucht, einige zentrale Kontinuitäten und Diskontinuitäten in Carnaps Psychologiebezug zwischen 1928 und 1932 herauszuarbeiten. Ich habe argumentiert, dass Carnaps Psychologiebezug im Aufbau einerseits eher oberflächlich ist (er beruft sich auf die Gestaltpsychologie, nimmt aber deren empirische Befunde nicht zur Kenntnis, sondern wählt stattdessen seine Methode der Quasizerlegung), er aber andererseits bereits hier bestimmte Grundannahmen psychophysikalischer Forschung implizit anerkennt, nämlich wenn er (in den $\S \S 57$ und 58) andeutet, wie eine „fremdpsychologische“ Methode im Zuge der Physikalisierung ihres Vokabulars vorgeht.

In Carnaps Schrift zur Psychologie in physikalischer Sprache tritt der zweite Aspekt noch deutlicher zutage: Seine hier vorgeschlagene Methode der Übersetzung psychologischer Sätze in die einer physikalischen Sprache spiegelt eins zu eins genau die Methode wider, die von Psychologen in der psychophysischen Tradition (zu der auch die Gestaltpsychologie zu zählen ist) praktiziert wird. Zugleich deutet sein Schlagabtausch mit Duncker darauf hin, dass die besagte Parallele für sein eigenes philosophisches Projekt irrelevant war. Dennoch, so habe ich argumentiert, ist es unwahrscheinlich, dass Carnaps selektive Anleihen bei der experimentellen Psychologie seiner Zeit gänzlich zufällig sind. So halte ich es für wahrscheinlich, dass ihm das methodische Vorgehen der Psychophysik seiner Zeit bekannt war.

Zusätzlich zu dieser empirischen Frage stellt sich - wie oben angedeutet - die normative Frage, ob Carnaps inkonsistente Bezugnahme auf die Psychologie seinem Projekt abträglich ist. An diesem Punkt möchte ich mich des Urteils enthalten, weise jedoch darauf hin, dass die Frage, wie eine sich selbst als ,wissenschaftlich“ verstehende Philosophie auf Theorien und Methoden der Wissenschaften Bezug nehmen sollte, nach wie vor aktuell ist.

\section{Literatur}

Ash, M. 1995. Gestalt psychology in German culture, 1890-1967: Holism and the quest for objectivity. Cambridge: Cambridge University Press.

Bühler, K. 1926. Die Krise der Psychologie. Kant-Stud 31(1-3): 455-526. 1927. Die Krise der Psychologie. Jena: Gustav Fischer. 
Carnap, R. 1923. Quasizerlegung: Ein Verfahren zur Ordnung nichthomogener Mengen mit den Mitteln der Beziehungslehre (Untertitel ,[MS: 27.12.22-25.1.23]“). Pittsburgh: University of Pittsburgh, Archives for Scientific Philosophy.

. 1928/1979. Der logische Aufbau der Welt. Frankfurt a. M./Berlin: Ullstein.

. 1931a. Überwindung der Metaphysik durch logische Analyse der Sprache. Erkenntnis 2: 219-241.

. 1931b. Die physikalische Sprache als Universalsprache der Wissenschaft. Erkenntnis 2: 432-465.

. 1932a. Psychologie in physikalischer Sprache. Erkenntnis 3:107-142.

1932b. Erwiderung auf die vorstehenden Aufsätze von E. Zilsel und K. Duncker. Erkenntnis 3:177-188.

Carus, A. 2016. Carnap and phenomenology: What happened in 1924? In Influences on the Aufbau, Hrsg. C. Damböck, 137-162. Dordrecht: Springer.

Damböck, C. 2017. ,Deutscher Empirismus': Studien zur Philosophie im deutschsprachigen Raum 1830-1930 (Veröffentlichungen des Instituts Wiener Kreis). Dordrecht: Springer.

Duncker, K. 1932. Behaviorismus und Gestaltpsychologie (Kritische Bemerkungen zu Carnaps „Psychologie in physikalischer Sprache“). Erkenntnis 3:162-176

Fechner, G.T. 1860. Elemente der Psychophysik. Leipzig: Breitkopf und Härtel.

Feest, U. 2007. Science and experience/Science of experience: Gestalt psychology and the antimetaphysical project of the Aufbau. Perspectives on Science 15(1): 38-62.

- 2017. Physicalism, introspection, and psychophysics: The Carnap/Duncker exchange. In Oppure si muove: Doing history and philosophy of science with Peter Machamer, The Western Ontario series in philosophy of science, Hrsg. M. Adams, Z. Biener, U. Feest und J. Sullivan, 113-125. Dordrecht: Springer.

Friedman, M. 1999. Epistemology in the Aufbau. In: ders., Reconsidering logical positivism, 114-151. Cambridge: Cambridge University Press. (Urspr.: Synthese 93(1-2):15-57 (1992)).

Heidelberger, M. 2003. The mind-body problem in the origin of logical empiricism: Feigl and psychophysical parallelism. In Logical empiricism: Historical and contemporary perspectives, Hrsg. P. Parrini, M. Salmon und W. Salmon, 233-262. Pittsburgh: Pittsburgh University Press.

Husserl, E. 1913/2009. Ideen zu einer reinen Phänomenologie und phänomenologischen Philosophie (Philosophische Bibliothek). Hamburg: Meiner.

Neurath, O. 1932. Protokollsätze. Erkenntnis 3:204-214.

Schlick, M. 2008. Allgemeine Erkenntnislehre (Moritz Schlick. Gesamtausgabe, Bd I/1), Hrsg. H. J. Wendel und F. O. Engler. Wien/New York: Springer. [first published 1918].

Sturm, T. 2012. Bühler and Popper: Kantian therapies for the crisis in psychology. Studies in History and Philosophy of Biological and Biomedical Science 43(2): 462-472.

Uebel, T. 2007. Empiricism at the crossroads: The Vienna Circle's protocol-sentence debate. Chicago: Open Court.

Zahavi, D. 2011. Der Sinn der Phänomenologie: Eine methodologische Reflexion. In Phänomenologie der Sinnereignisse, Hrsg. H.-D. Gondek, T.N. Klass und L. Tengelyi, 101-119. München: Wilhelm Fink.

Open Access This chapter is licensed under the terms of the Creative Commons Attribution 4.0 International License (http://creativecommons.org/licenses/by/4.0/), which permits use, sharing, adaptation, distribution and reproduction in any medium or format, as long as you give appropriate credit to the original author(s) and the source, provide a link to the Creative Commons license and indicate if changes were made.

The images or other third party material in this chapter are included in the chapter's Creative Commons license, unless indicated otherwise in a credit line to the material. If material is not included in the chapter's Creative Commons license and your intended use is not permitted by statutory regulation or exceeds the permitted use, you will need to obtain permission directly from the copyright holder. 